\title{
Protecting a spin ensemble against decoherence in the strong-coupling regime of cavity QED
}

\author{
S. Putz ${ }^{1,2 \dagger}$, D. O. Krimer ${ }^{3 \dagger}$, R. Amsüss ${ }^{1}$, A. Valookaran ${ }^{1}$, T. Nöbauer ${ }^{1,4}$, J. Schmiedmayer ${ }^{1}$, S. Rotter ${ }^{3 \star}$ \\ and J. Majer ${ }^{1,2 \star}$
}

Hybrid quantum systems based on spin ensembles coupled to superconducting microwave cavities are promising candidates for robust experiments in cavity quantum electrodynamics (QED) and for future technologies employing quantum mechanical effects ${ }^{1-4}$. At present, the main source of decoherence in these systems is inhomogeneous spin broadening, which limits their performance for the coherent transfer and storage of quantum information ${ }^{5-7}$. Here we study the dynamics of a superconducting cavity strongly coupled to an ensemble of nitrogen-vacancy centres in diamond. We experimentally observe how decoherence induced by inhomogeneous broadening can be suppressed in the strong-coupling regime-a phenomenon known as 'cavity protection' ${ }^{157}$. To demonstrate the potential of this effect for coherent-control schemes, we show how appropriately chosen microwave pulses can increase the amplitude of coherent oscillations between the cavity and spin ensemble by two orders of magnitude.

The processing of quantum information requires special devices that can store and manipulate quantum bits. Hybrid quantum systems ${ }^{2}$ combine the advantages of different systems to overcome their individual physical limitations. In this context superconducting microwave cavities have emerged as ideal tools for realizing strong coupling to qubits ${ }^{3,4,8-12}$ for the transfer of excitations on the singlephoton level ${ }^{13,14}$. For the storage of quantum information the negatively charged nitrogen-vacancy (NV) centres in diamond show great potential, especially owing to their long coherence times (up to one second $\mathrm{d}^{15}$ ) and to the combination of microwave and optical transitions which makes them an easily accessible and controllable qubit $^{16}$. Coherently passing quantum information between such a spin and a cavity requires that they are strongly coupled to each other. As has recently been shown ${ }^{4,10-12}$, this limit can be reached by collective coupling to a large spin ensemble, in which case the coupling strength is increased by the square root of the ensemble size. However, this collective coupling comes with a considerable downside: in a solid-state environment a spin is always prone to inhomogeneous broadening. In particular, for an ensemble of NV centres, magnetic dipolar interaction with excess nuclear and electron spins in the diamond crystal leads to an inhomogeneous broadening of the spin transition ${ }^{17}$, which acts as the dominant source of decoherence. Several approaches, including echo-type refocusing techniques ${ }^{18,19}$, have been suggested to overcome this limitation. Here we will concentrate on recent theoretical proposals which rely on the specific shape of the inhomogeneous spectral

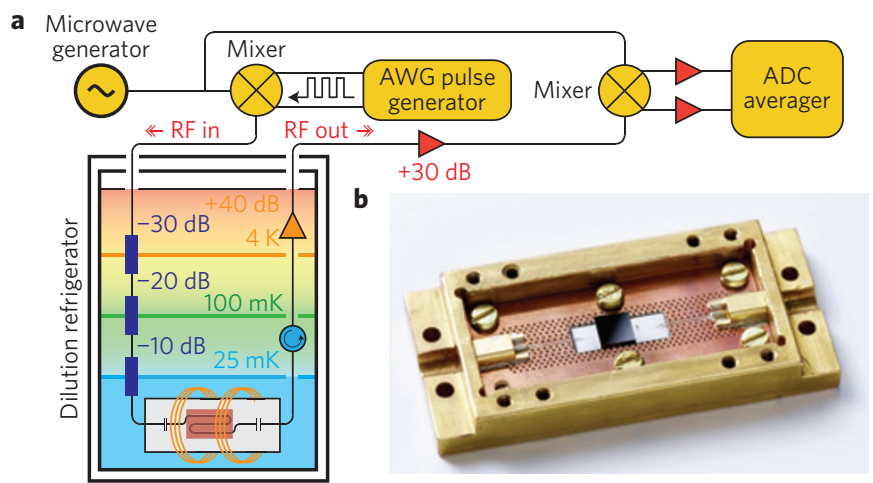

Figure 1 | Experimental set-up. a, The superconducting coplanar waveguide resonator with the diamond on top is cooled to $\sim 25 \mathrm{mK}$ in a dilution refrigerator. In our homodyne detection measurements, the input microwave signal is split into two paths, both serving as a reference signal as well as for testing and controlling our experiment. Outside the cryostat both signal paths are combined by a frequency mixer and the quadratures I and $\mathrm{Q}$ are recorded with a fast analog-to-digital converter (ADC) with subnanosecond time resolution. AWG: arbitrary waveform generator. b, Photograph of a superconducting microwave cavity with an enhanced neutron-irradiated type lb synthetic diamond (black) on top, encased in a gold-plated copper sample box.

spin distribution $\rho(\omega)$ of the NV centre ensemble. In our explicitly time-dependent study we will demonstrate the so-called 'cavityprotection effect ${ }^{5,7}$, which was predicted in these proposals but has remained so far unobserved.

Our experiment is performed in a standard dilution refrigerator with the corresponding set-up being sketched in Fig. 1a and a photograph of the resonator with a synthetic diamond on top shown in Fig. 1b. To avoid thermal excitations we cool the entire set-up to a temperature of $25 \mathrm{mK}$, where the estimated thermal spin polarization is $99 \%$. By applying an external magnetic field $|\mathbf{B}|=9.4 \mathrm{mT}$ through a set of two superconducting Helmholtz coils we Zeeman tune the NV spin ensemble into resonance with the cavity. Our resonator has a fundamental resonance at $\omega_{c} / 2 \pi=2.6899 \mathrm{GHz}$ with a quality factor of $Q=3,060$. To excite and probe the coupled system we inject microwave pulses into the cavity and perform time-resolved transmission spectroscopy by a fast

\footnotetext{
${ }^{1}$ Vienna Center for Quantum Science and Technology, Atominstitut, Vienna University of Technology, Stadionallee 2, 1020 Vienna, Austria, ${ }^{2}$ Zentrum für Mikro- und Nanostrukturen, Vienna University of Technology, Floragasse 7, 1040 Vienna, Austria, ${ }^{3}$ Institute for Theoretical Physics, Vienna University of Technology, Wiedner Hauptstrasse 8-10/136, 1040 Vienna, Austria, ${ }^{4}$ Research Institute of Molecular Pathology and Max F. Perutz Laboratories, University of Vienna, Dr.-Bohr-Gasse 7, 1030 Vienna, Austria. These authors contributed equally to this work.

*e-mail: stefan.rotter@tuwien.ac.at; johannes@majer.ch
} 
a

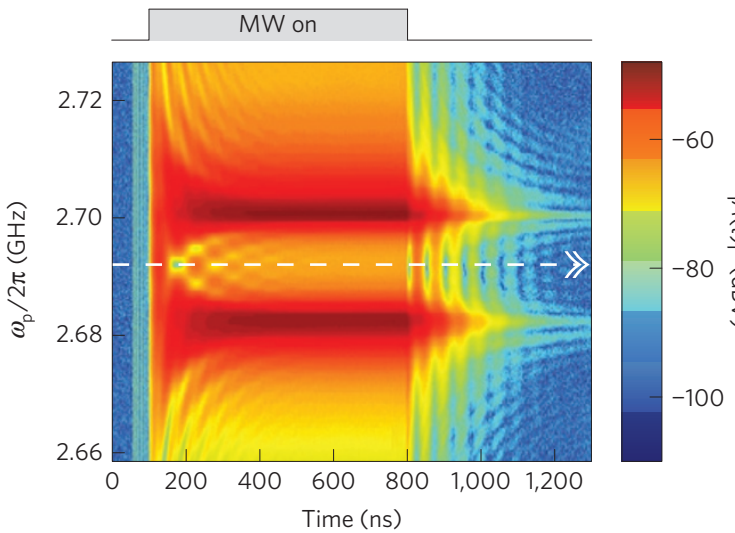

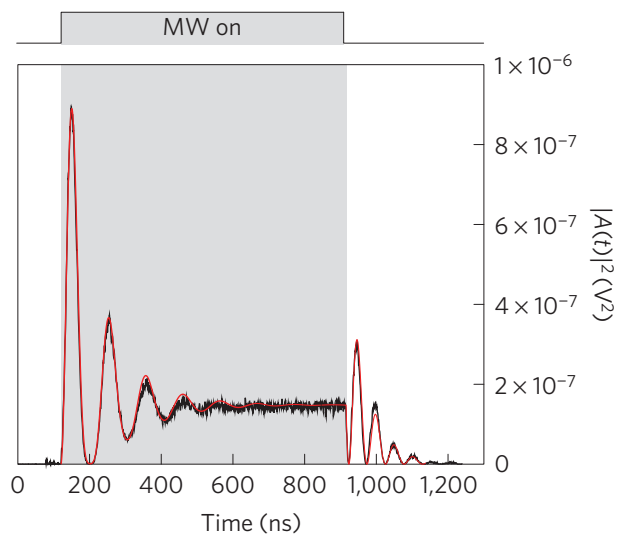

Figure $\mathbf{2}$ | Time domain measurements of the cavity transmission. a, Transmission of a rectangular microwave (MW) pulse through the cavity versus time and probe frequency $\omega_{\mathrm{p}}$ (the spins are in resonance with the cavity, $\omega_{\mathrm{s}}=\omega_{\mathrm{c}}$ ). The observation of strong mode-splitting into the two hybridized modes $\left|\Psi_{ \pm}\right\rangle \approx \frac{1}{\sqrt{2}}\left(|0\rangle_{c}|1\rangle_{s} \pm|1\rangle_{c}|0\rangle_{S}\right.$ ) (see dark red enhancements split by $\Omega_{R} / 2 \pi=19.2 \mathrm{MHz}$ ) confirms that the system is deep in the strong-coupling regime. $\mathbf{b}$, The dynamics at the resonant probe frequency $\omega_{\mathrm{p}}=\omega_{\mathrm{s}}=\omega_{\mathrm{c}}$ (white dashed line in $\mathbf{a}$ ) is compared with the theoretical prediction for the cavity probability amplitude $|A(t)|^{2}$ (experiment: black: theory: red). Excellent agreement is achieved when incorporating the correct non-Lorentzian spectral spin distribution. After switching on the pulse, the system exhibits damped Rabi oscillations, with frequency $\Omega_{\mathrm{R}}$, that equilibrate at a stationary state. After switching off the pulse, the cavity amplitude first decays from the stationary state and then features a pronounced overshoot corresponding to a strongly non-Markovian release of the energy stored in the spin ensemble back into the cavity.

homodyne detection set-up with subnanosecond time resolution. The number of microwave photons in the cavity remains at or below $\sim 10^{6}$, which is very low compared with the number of $\sim 10^{12}$ NV spins involved in the coupling, ensuring that the HolsteinPrimakoff ${ }^{20}$ approximation is valid for describing our experiments. As a result of this approximation, the excitations in the system can be treated as non-interacting quasiparticles ${ }^{5}$ and the dynamics for the $\sim 10^{6}$ photons in our system is equivalent to that of a corresponding single-photon experiment as relevant for quantum information processing (see Supplementary Methods Section II).

Our starting point to account for the dynamics of a singlemode cavity coupled to a spin ensemble is the Tavis-Cummings Hamiltonian $^{21}$, which reads in the rotating wave approximation

$$
\begin{aligned}
H=\hbar \omega_{c} a^{\dagger} a+ & \frac{\hbar}{2} \sum_{j}^{N} \omega_{j} \sigma_{j}^{z}+i \hbar \sum_{j}^{N}\left[g_{j} \sigma_{j}^{-} a^{\dagger}-g_{j}^{*} \sigma_{j}^{+} a\right] \\
& -i \hbar\left[\eta(t) a^{\dagger} \mathrm{e}^{-i \omega_{p} t}-\eta(t)^{*} a \mathrm{e}^{i \omega_{p} t}\right]
\end{aligned}
$$

where $a^{\dagger}$ and $a$ are standard creation and annihilation operators of the cavity mode and $\sigma_{j}^{ \pm}, \sigma_{j}^{z}$ are the Pauli operators associated with each individual spin. The first and second terms stand for the uncoupled resonator with frequency $\omega_{c}$ and for the spin ensemble with frequencies $\omega_{j}$, centred around $\omega_{s}$, respectively. The third and the last terms describe the cavity-spin interaction with coupling strength $g_{j}$ as well as the driving electromagnetic field injected into the cavity with amplitude $\eta(t)$ and frequency $\omega_{\mathrm{p}}$. The collective coupling to a large number of spins allows us to enter the strong-coupling regime of QED, for which the interaction term is commonly reduced to a collective term ${ }^{22} \Omega\left(S^{-} a^{\dagger}-S^{+} a\right)$, where the collective spin operators read $S^{ \pm}=\frac{1}{\sqrt{N}} \sum_{j}^{N} \sigma_{j}^{ \pm}$. The prefactor $\Omega^{2}=\sum_{j}^{N} g_{j}^{2}$ stands for an effective coupling strength, which scales up a single cavity-spin interaction, typically on the order of $g_{j} / 2 \pi \sim 12 \mathrm{~Hz}$, by a factor $\sqrt{N}$ (refs 3,4,23). In this formulation the effective spin waves that are excited by the cavity mode can be identified as superradiant collective Dicke states which are effectively damped by the coupling to subradiant states in the ensemble ${ }^{13,24}$. To accurately describe the corresponding dynamics we also need to take into account the specific profile of the spectral spin distribution ${ }^{7} \rho(\omega)=\sum_{j} g_{j}^{2} \delta\left(\omega-\omega_{j}\right) / \Omega^{2}$. We achieve this by setting up a Volterra integral equation (see Supplementary Methods), $A(t)=\int_{0}^{t} \mathrm{~d} \tau \int \mathrm{d} \omega \mathcal{K}(\rho(\omega) ; t-\tau) A(\tau)+\mathcal{F}(t)$, for the cavity amplitude $A(t)=\langle a(t)\rangle$. This includes a memory-kernel $\mathcal{K}(t-\tau)$, responsible for the non-Markovian feedback of the NV ensemble on the cavity, and the function $\mathcal{F}(t)$, which describes the contribution from an external drive and from the initial spin excitation. In the following, the cavity amplitude $|A(t)|^{2}$, calculated with this approach for stationary and pulsed driving fields, will be compared to its experimental counterpart-that is, the timeresolved microwave intensity measured in transmission through the cavity.

First, to demonstrate that our experiment is in the strongcoupling regime (having $\omega_{\mathrm{s}}=\omega_{\mathrm{c}}$ ) we apply a rectangular microwave pulse which is sufficiently long compared with the cavity decay rate $\kappa$, total decoherence rate $\Gamma$ and coupling strength $\Omega(800 \mathrm{~ns} \gg 1 / 2 \kappa=199 \mathrm{~ns}, 1 / \Gamma=53 \mathrm{~ns}, \pi / \Omega=58 \mathrm{~ns})$ to drive the system into a steady state with varying probe frequency $\omega_{\mathrm{p}}$. Figure $2 \mathrm{a}$ shows that two effective eigenstates (polaritons) of the coupled system emerge in the transmission, $\left|\Psi_{ \pm}\right\rangle \approx \frac{1}{\sqrt{2}}\left(|0\rangle_{c}|1\rangle_{s} \pm|1\rangle_{c}|0\rangle_{s}\right)$, corresponding to the symmetric and antisymmetric superposition of the cavity and spin eigenstates, respectively. Strong coupling is secured because the Rabi splitting between these states $\Omega_{\mathrm{R}} / 2 \pi=19.2 \mathrm{MHz}$ is substantially larger than the total decay rate of the system $\Gamma / 2 \pi=3.0 \mathrm{MHz}$ (full-width at half-maximum). The latter consists of a cavity decay rate, $\kappa / 2 \pi=0.4 \mathrm{MHz}$ (half-width at half-maximum), as well as of a spin decay rate which contains a negligibly small spin dissipation $\gamma \rightarrow 0$ and a dominant contribution from the inhomogeneous broadening of the spin ensemble. Detailed spectroscopic measurements of the stationary transmission ${ }^{6}$ reveal that the spectral function $\rho(\omega)$ which accurately captures the broadening is neither Lorentzian nor Gaussian, but has the intermediate form of a $q$-Gaussian ${ }^{6}$ (see Supplementary Methods Section I). As shown in Fig. 2b, our explicitly time-dependent theoretical description yields excellent quantitative agreement with the experimental data, using such a $q$-Gaussian distribution function with a linewidth of $\gamma_{q} / 2 \pi=9.4 \mathrm{MHz}$ (full-width at half-maximum), a shape parameter $q=1.39$ and an effective coupling strength $2 \cdot \Omega / 2 \pi=17.2 \mathrm{MHz}$. After turning on and switching off the microwave pulse, coherent Rabi oscillations occur between the cavity and the spin ensemble, which we reproduce accurately, including their damping. Interestingly, the first Rabi 


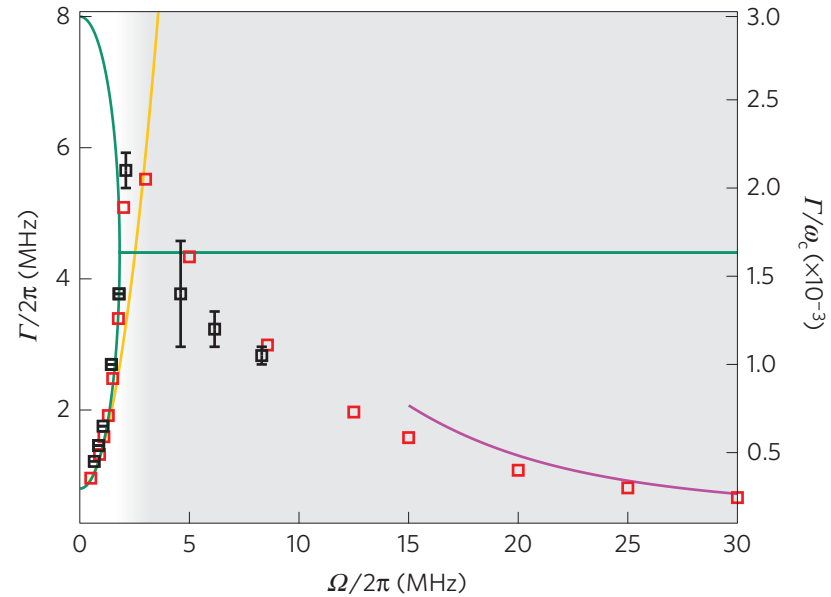

Figure 3 | Characterization of the decay dynamics as a function of coupling strength. For weak coupling, the decay rate $\Gamma$ of the cavity probability amplitude, $|A(t)|^{2}$, increases as a function of the coupling strength $\Omega$. For strong coupling, this trend reverses, showing a protection of the system against decoherence. Black symbols: experimentally observed decay rates from the steady state. Red symbols: decay rates extracted from the full numerical calculations. Orange curve: decay rate,

$\Gamma=2\left[\kappa+\pi \Omega^{2} \rho\left(\omega_{\mathrm{s}}\right)\right]$, derived under the Markovian approximation. Green curve: characteristic decay rates, $\Gamma_{1,2}=\Delta+\kappa \pm \sqrt{(\Delta-\kappa)^{2}-4 \Omega^{2}}$ under the assumption of a Lorentzian distribution of the spin density. Magenta curve: analytical estimate of $\Gamma=\kappa+\pi \Omega^{2} \rho\left(\omega_{\mathrm{s}} \pm \Omega\right)$ in the very strong-coupling regime. The background colour indicates at which coupling strength the system undergoes a transition from the Markovian (white) to the non-Markovian (grey) regime. The error bars correspond to the minimal and maximal values of the estimated decay rates from the experimental data. As no Rabi oscillations occur in the Markovian regime, the values for the decay rate can be determined more accurately in this limit.

peak shows a pronounced overshoot after switching off the microwave drive, at which the energy stored in the spin ensemble is coherently released back into the cavity. These oscillations are a hallmark of the non-Markovian character of the system dynamics in the strong-coupling regime, for which an accurate knowledge of the memory-kernel $\mathcal{K}(t-\tau)$ in our Volterra equation is essential.

Our hybrid cavity-spin system can not be modelled by two coupled damped harmonic oscillators as in the case of a purely Lorentzian spin distribution. The spectral profile of a $q$-Gaussian has marked consequences on the dynamics in the strong-coupling regime: in particular, for spectral distributions $\rho(\omega)$ with tails that fall off faster than a Lorentzian lineshape $\left(\propto 1 / \omega^{2}\right)$, an increasing coupling strength was predicted to lead to a reduction of the decay rate $\Gamma$ and to protect the system against decoherencehence the name 'cavity-protection effect' ${ }^{5,7}$. In a nutshell, 'cavity protection' can be understood as follows: in the strong-coupling regime, the cavity couples to a set of superradiant states forming hybridized energy levels split by $\hbar \Omega_{\mathrm{R}}$. The resulting polariton states are coupled to subradiant spin-wave modes acting as the main source of decoherence, and the total decay rate depends on the energetic gap between the polaritons and the subradiant states. If the spectral profile of the inhomogeneous spin distribution decays sufficiently fast for increasing gap size, an energetic decoupling of the superradiant polaritons from the subradiant spin-wave modes occurs, leading to a suppressed damping of the polaritons. Although the origin of this intriguing effect can also be understood on the basis of an ensemble of coupled classical oscillators ${ }^{5,7}$, the important point to note is that it also survives on the level of single-photon excitations (see Supplementary Methods Section II).
As the tails of our spin distribution satisfy the required fast decay, we now have the possibility to probe this exceptional behaviour in the experiment. We measure the decay rate $\Gamma(\Omega)$ of the cavity amplitude after driving the system for different coupling strengths $\Omega$ into steady state using a sufficiently long rectangular pulse (as in Fig. 2b, see Methods). The values of $\Gamma(\Omega)$, as determined by the slope of the temporal decay after switch off, are shown in Fig. 3. We find the decay rate to vary over almost one order of magnitude in a strongly non-monotonic fashion: in the weakcoupling regime the decay rate $\Gamma$ increases with growing coupling strength $\Omega$ as a result of the Purcell effect ${ }^{25}$ as the cavity mode increasingly couples to the spin ensemble. Entering the strongcoupling regime, this trend reverses and $\Gamma$ decreases with growing $\Omega$. To highlight this remarkable phenomenon, we also plot in Fig. 3 the behaviour for a Lorentzian spin distribution, for which $\Gamma(\Omega)$ is constant in the strong-coupling limit. Performing a Laplace transform of our Volterra equation we find that in the limit of very strong coupling $(\Omega \rightarrow \infty)$ the decay rate takes the following closed analytical form $\Gamma=\kappa+\pi \Omega^{2} \rho\left(\omega_{\mathrm{s}} \pm \Omega\right.$ ) (in agreement with a stationary analysis ${ }^{7}$ ). Whereas the maximally reachable value of $\Omega / 2 \pi=8.6 \mathrm{MHz}$ in our device already leads to a considerable reduction of $\Gamma$ by $50 \%$ below its maximum, our numerical results (Fig. 3) suggest a further reduction of the decay rate with increasing coupling strength by an order of magnitude. We predict that a three times higher $\Omega$ could be achieved by filling up the cavity with diamond and by further increasing the NV density. Having the mode volume entirely occupied with NV centres, a factor of two enhanced NV concentration would already bring us close to exploiting the full potential of the cavity-protection effect. We estimate that such an increased NV concentration would cause a mean NV-NV interaction strength of $120 \mathrm{kHz}$, which is still small enough to have only a negligible influence on the dynamics of our system.

In a next step, we demonstrate that the 'cavity-protection effect' can also be employed for the realization of coherentcontrol schemes. In particular, we address a central question when dealing with coherently driven spin ensembles-namely, how to achieve high excitation levels in the spin ensemble with limited driving powers ${ }^{18,26}$. In a simplified picture of two coupled harmonic oscillators this can be achieved by a drive modulated with the inverse of the effective coupling strength. To realize this for the non-Lorentzian spectral spin distribution of our ensemble a pulsed driving is required to match the Rabi frequency $\Omega_{\mathrm{R}}$ rather than the effective coupling strength $2 \Omega$, which quantities are here quite different from each other. We thus probe our set-up by a driving field with a carrier frequency $\omega_{\mathrm{p}}=\omega_{\mathrm{c}}=\omega_{\mathrm{s}}$ and a periodic modulation with tunable period $\tau$. Realizing the latter with a simple periodic sign-change of the carrier signal, we find that this driving scheme produces giant oscillations in the transmission (Fig. 4a) corresponding to a coherent exchange of energy between the cavity and the spin ensemble. A maximum oscillation amplitude occurs exactly at the point where the modulation period $\tau$ coincides with the inverse of the Rabi splitting $2 \pi / \Omega_{\mathrm{R}}$. Note that at this resonant driving the steady-state oscillation amplitude in the transmission signal (Fig. 4b) exceeds the stationary amplitude (Fig. 2b) by two orders of magnitude, although the net power applied to the cavity is exactly the same in both cases. Our approach demonstrates how to sustain coherent oscillations and how to reach considerably high excitation amplitudes of the spin ensemble without using strong driving powers. For comparison, we also plot in Fig. $4 \mathrm{~b}$ the results both for a $q$-Gaussian as well as for a Lorentzian spin density, which clearly shows the substantially lower excitation amplitudes for the Lorentzian case. This clear signature of the 'cavity-protection effect' paves the way for the realization of sophisticated coherent-control schemes in the strong-coupling regime of QED. 
a
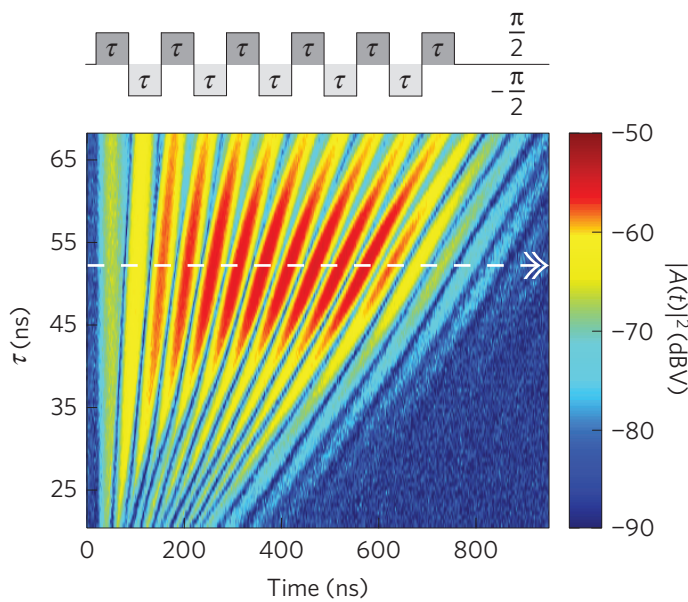

b
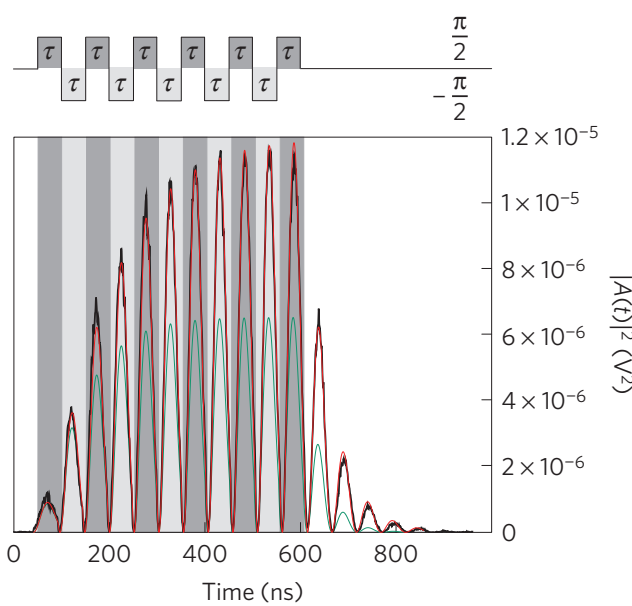

Figure 4 | Enhancement of the cavity transmission intensity by pulsed driving. a, The cavity transmission of eleven successive rectangular microwave pulses with carrier frequency $\omega_{\mathrm{p}}=\omega_{\mathrm{c}}=\omega_{\mathrm{s}}$, phase-switched by $\pi$, as a function of time and pulse duration $\tau$ (see top panel for the pulse shape). b. Dynamics at the largest enhancement of the cavity transmission, corresponding to a pulse duration of $\tau=52$ ns (indicated by the white dashed line in $\mathbf{a}$ ) equal to the Rabi period $T_{R}=2 \pi / \Omega_{R}$. After switching off the probe signal the system settles back to the ground state through damped Rabi oscillations. Excellent agreement between experiment (black curve) and theory (red curve) is found, using the same system parameters as in Fig. 3. A Lorentzian spin distribution in the theoretical calculations (green curve) leads to a considerably smaller enhancement owing to the absence of the cavity-protection effect.

\section{Methods}

The cavity is an overcoupled $\lambda / 2$ resonator, made out of $200 \mathrm{~nm}$ thick sputtered niobium on a $330 \mu \mathrm{m}$ thick sapphire substrate, structured by optical lithography and reactive ion etching. The diamond sample is a commercially available type Ib high-pressure high-temperature diamond (HPHT) with a size of $4.5 \times 2.25 \times 0.5 \mathrm{~mm}^{3}$ and two polished (100) surfaces. The crystal contains an initial concentration of $200 \mathrm{ppm}$ nitrogen and has a natural abundance of ${ }^{13} \mathrm{C}$ nuclear isotopes. We achieve a total density of $\sim 6 \mathrm{ppm} \mathrm{NV}$ centres by $50 \mathrm{~h}$ of neutron irradiation with a fluence of $5 \times 10^{17} \mathrm{~cm}^{-2}$ and by annealing the crystal for $3 \mathrm{~h}$ at $900^{\circ} \mathrm{C}$, resulting in a conversion efficiency from initial nitrogen to $\mathrm{NV}$ centres of 3\%. The diamond sample has been characterized by means of a room-temperature confocal laser scanning microscope, allowing us to measure the NV density and the zero-field splitting parameters $D$ and $E$ by optically detected magnetic resonance ${ }^{27}$. Residual nitrogen is incorporated in the diamond mainly as substitutional single defect centres, which also act as paramagnetic impurities with $S=1 / 2$ and thus form the main source of inhomogeneous broadening.

The NV centre is a paramagnetic impurity which consists of a substitutional nitrogen atom and an adjacent vacancy in the diamond lattice. The energy level structure features an electron spin triplet $(S=1)$ as its ground state ${ }^{28}$. Owing to negligible direct NV-NV interactions, it can be described, to lowest order, by the Hamiltonian $^{29}, H / h=D S_{z}^{2}+E\left(S_{\mathrm{x}}^{2}-S_{\mathrm{y}}^{2}\right)+\mu \mathbf{B S}$ with $\mathbf{S}=\left(S_{x}, S_{y}, S_{z}\right), D=2.877 \mathrm{GHz}$, $E=7.7 \mathrm{MHz}$ and $\mu=28 \mathrm{MHz} \mathrm{mT}^{-1}$, providing us with an estimate for the mean spin transition frequency $\omega_{\mathrm{s}}$. The zero-field splitting term $D=2.877 \mathrm{GHz}$ corresponds to $138 \mathrm{mK}$, which is high compared with the temperature of $25 \mathrm{mK}$ at which the experiments are performed and allows us to thermally polarize the NV spins up to $99 \%$. In the diamond lattice four crystallographic orientations of the NV defect are possible. Our diamond sample has a (100) surface orientation. We apply a d.c. magnetic field of $9.4 \mathrm{mT}$ to Zeeman tune the spins into resonance with the cavity, which is applied in the plane of the resonator and therefore in the (100) plane of the crystal. We rotate the magnetic field direction by $22.5^{\circ}$ in the plane, at which only two subensembles are degenerate. The external magnetic field leads, on the one hand, to a Zeeman splitting of subensembles such that the maximal possible ensemble-cavity coupling strength is reduced by a factor of $\sqrt{2}$. On the other hand, it narrows the width of the spectral spin distribution induced by a misalignment of the applied external magnetic field with respect to the crystallographic frame.

For the application of short microwave pulses with adjustable phase and power we use a frequency mixer controlled by an arbitrary waveform generator (AWG). The microwave signal passes the cryostat and is attenuated by $-60 \mathrm{~dB}$ when reaching the resonator. The transmitted signal is fed into a circulator and amplified on the $4 \mathrm{~K}$ stage. Using a homodyne detection scheme, the transmitted and amplified microwave signal is mixed with the reference signal and both quadrature signals I and Q are recorded by a fast analog-to-digital converter with $2 \mathrm{GS} \mathrm{s}^{-1}$ sampling frequency. The AWG repeats the pulse sequence and $10^{6}$ single traces are averaged. From the two quadratures I and Q the transmitted microwave intensity $|A(t)|^{2}$ is calculated and plotted in Figs 2 and 4 . Voltage fluctuations give a standard deviation of $\pm 1.02 \times 10^{-5}(\mathrm{~V})$ on the quadratures I and Q. The squared steady-state amplitude of the cavity transmission (Fig. 2b) gives a mean signal of $|A|^{2}=1.44 \times 10^{-7} \pm 6.85 \times 10^{-9}\left(\mathrm{~V}^{2}\right)$. To reduce the coupling strength (Fig. 3) we repeatedly pump the resonator with high power and a long microwave pulse, corresponding to $\sim 2.5 \times 10^{7}$ microwave photons in the cavity. For this measurement power, a non-negligible number of NV spins gets excited, leading to a reduced number of ground-state spins coupled to the cavity.

Received 29 March 2014; accepted 30 June 2014; published online 17 August 2014

\section{References}

1. Zhu, X. et al. Coherent coupling of a superconducting flux qubit to an electron spin ensemble in diamond. Nature 478, 221-224 (2011).

2. Xiang, Z-L., Ashhab, S., You, J. Q. \& Nori, F. Hybrid quantum circuits: Superconducting circuits interacting with other quantum systems. Rev. Mod. Phys. 85, 623-653 (2013).

3. Kubo, Y. et al. Hybrid quantum circuit with a superconducting qubit coupled to a spin ensemble. Phys. Rev. Lett. 107, 220501 (2011).

4. Amsüss, R. et al. Cavity QED with magnetically coupled collective spin states. Phys. Rev. Lett. 107, 060502 (2011).

5. Kurucz, Z., Wesenberg, J. H. \& Mølmer, K. Spectroscopic properties of inhomogeneously broadened spin ensembles in a cavity. Phys. Rev. A 83, 053852 (2011).

6. Sandner, K. et al. Strong magnetic coupling of an inhomogeneous nitrogen-vacancy ensemble to a cavity. Phys. Rev. A 85, 053806 (2012).

7. Diniz, I. et al. Strongly coupling a cavity to inhomogeneous ensembles of emitters: Potential for long-lived solid-state quantum memories. Phys. Rev. A 84, 063810 (2011).

8. Wallraff, A. et al. Strong coupling of a single photon to a superconducting qubit using circuit quantum electrodynamics. Nature 431, 162-167 (2004).

9. Majer, J. Coupling superconducting qubits via a cavity bus. Nature 449, 443-447 (2007).

10. Kubo, Y. et al. Strong coupling of a spin ensemble to a superconducting resonator. Phys. Rev. Lett. 105, 140502 (2010).

11. Schuster, D. I. et al. High-cooperativity coupling of electron-spin ensembles to superconducting cavities. Phys. Rev. Lett. 105, 140501 (2010).

12. Probst, S. et al. Anisotropic rare-earth spin ensemble strongly coupled to a superconducting resonator. Phys. Rev. Lett. 110, 157001 (2013).

13. Kubo, Y. et al. Storage and retrieval of a microwave field in a spin ensemble. Phys. Rev. A 85, 012333 (2012).

14. Saito, S. et al. Towards realizing a quantum memory for a superconducting qubit: Storage and retrieval of quantum states. Phys. Rev. Lett. 111, 107008 (2013).

15. Bar-Gill, N., Pham, L., Jarmola, A., Budker, D. \& Walsworth, R. Solid-state electronic spin coherence time approaching one second. Nature Commun. 4, 456 (2013). 
16. Childress, L. et al. Coherent dynamics of coupled electron and nuclear spin qubits in diamond. Science 314, 281-285 (2006).

17. Stanwix, P. L. et al. Coherence of nitrogen-vacancy electronic spin ensembles in diamond. Phys. Rev. B 82, 201201 (2010).

18. Grezes, C. et al. Multimode storage and retrieval of microwave fields in a spin ensemble. Phys. Rev. X 4, 021049 (2014).

19. $\mathrm{Wu}, \mathrm{H}$. Storage of multiple coherent microwave excitations in an electron spin ensemble. Phys. Rev. Lett. 105, 140503 (2010).

20. Primakoff, H. \& Holstein, T. Many-body interactions in atomic and nuclear systems. Phys. Rev. 55, 1218-1234 (1939).

21. Tavis, M. \& Cummings, F. W. Exact solution for an $N$-molecule-radiation-field Hamiltonian. Phys. Rev. 170, 379-384 (1968).

22. Emary, C. \& Brandes, T. Chaos and the quantum phase transition in the Dicke model. Phys. Rev. E 67, 066203 (2003).

23. Verdú, J. et al. Strong magnetic coupling of an ultracold gas to a superconducting waveguide cavity. Phys. Rev. Lett. 103, 043603 (2009).

24. Dicke, R. H. Coherence in spontaneous radiation processes. Phys. Rev. 93, 99-110 (1954).

25. Proceedings of the American Physical Society. Phys. Rev. 69, 674 (1946).

26. Sigillito, A. J. et al. Fast, low-power manipulation of spin ensembles in superconducting microresonators. Appl. Phys. Lett. 104, 222407 (2014).

27. Nöbauer, T. et al. Creation of ensembles of nitrogen-vacancy centers in diamond by neutron and electron irradiation. Preprint at http://arxiv.org/abs/1309.0453 (2013).

28. Redman, D. A., Brown, S., Sands, R. H. \& Rand, S. C. Spin dynamics and electronic states of $\mathrm{N}-\mathrm{V}$ centers in diamond by EPR and four-wave-mixing spectroscopy. Phys. Rev. Lett. 67, 3420-3423 (1991).
29. Jelezko, F., Gaebel, T., Popa, I., Gruber, A. \& Wrachtrup, J. Observation of coherent oscillations in a single electron spin. Phys. Rev. Lett. 92, 076401 (2004)

\section{Acknowledgements}

We would like to thank C. Koller, F. Mintert, P. Rabl, H. Ritsch, K. Sandner and M. Trupke for helpful discussions, and D. Brasch/Terra Mater Magazin for the image shown in Fig. 1b. The experimental effort has been supported by the TOP grant of TU Vienna. S.P. acknowledges support by the Austrian Science Fund (FWF) in the framework of the Doctoral School 'Building Solids for Function' (Project W1243). D.O.K. and S.R. acknowledge funding by the FWF through Projects No. F25-P14 (SFB IR-ON) and No. F49-P10 (SFB NextLite).

\section{Author contributions}

S.P., R.A., A.V., T.N., J.S. and J.M. designed and set up the experiment. S.P., R.A. and A.V. carried out the measurements with supervision by J.M. D.O.K. and S.R. devised the theoretical framework and performed the calculations. S.P., D.O.K., S.R. and J.M. wrote the first manuscript draft, to which all authors suggested improvements.

\section{Additional information}

Supplementary information is available in the online version of the paper. Reprints and permissions information is available online at www.nature.com/reprints.

Correspondence and requests for materials should be addressed to S.R. or J.M.

\section{Competing financial interests}

The authors declare no competing financial interests. 